\title{
ARTíCULO \\ Preferencia y tolerancia térmica de juveniles de chita Anisotremus scapularis (Pisces: Haemulidae)
}

Thermal preference and tolerance of Peruvian grunt Anisotremus scapularis juveniles (Pisces: Haemulidae)

\section{Candy León-Palomino ${ }^{1,2 *}$, Jorge Flores-Mego ${ }^{2}$, Jhon Dionicio-Acedo ${ }^{2}$, Maryandrea Rosado-Salazar ${ }^{2}$, Jonathan Flye-Sainte-Marie ${ }^{3}$ y Arturo Aguirre-Velarde ${ }^{2}$}

${ }^{1}$ Facultad de Ciencias Biológicas, Universidad Nacional Mayor de San Marcos, Av. Venezuela cdra. 34, Av. Universitaria s/n. Lima, Perú.*candyleon5@gmail.com

${ }^{2}$ Laboratorio de Ecofisiología Acuática, Instituto del Mar del Perú (IMARPE), Esquina Gamarra y General Valle S/N Chucuito, Callao, Perú ${ }^{3}$ Laboratoire des Sciences de L'Environnement Marin- LEMAR, UMR 6539 (UBO/CNRS/IRD/Ifremer), Institut Universitaire Européen de la Mer (IUEM), Rue Dumont d'Urville, 29280 Plouzané, France

\begin{abstract}
Thermal preference and tolerance of juvenile Anisotremus scapularis (Pisces: Haemulidae) at different acclimation temperatures $\left(14,17\right.$ and $22^{\circ} \mathrm{C}$ ) were evaluated in order to estimate the optimum temperature and the thermal tolerance limits of this species. The preference was determined through acute preferred temperature. Maximum critical temperature and mean lethal temperature allowed to estimate thermal tolerance. The results show that acclimation temperatures higher than $14^{\circ} \mathrm{C}$ increase both thermal preference and tolerance. However, individuals acclimatized to $22^{\circ} \mathrm{C}$ preferred temperatures lower than their acclimation. In addition, thermal tolerance was identical between individuals acclimatized at $17^{\circ} \mathrm{C}$ and $22^{\circ} \mathrm{C}$. The optimum temperature of juvenile $\mathrm{A}$. scapularis was $18.7 \pm 2^{\circ} \mathrm{C}$ and its upper and lower tolerance limit was 32.6 and $10.1^{\circ} \mathrm{C}$, respectively. Based on the results, we concluded that a temperature of $18.7 \pm 2^{\circ} \mathrm{C}$ would optimize the physiological processes in juvenile $\mathrm{A}$. scapularis and that temperature higher than $32.6^{\circ} \mathrm{C}$ and lower than $10.1^{\circ} \mathrm{C}$ would cause the immediate death of the organism.
\end{abstract}

Key words: Anisotremus scapularis, thermal preference, thermal tolerance, acclimation temperature, optimum temperature

\begin{abstract}
Resumen.- Se evaluó la preferencia y tolerancia térmica de juveniles de Anisotremus scapularis (Pisces: Haemulidae) a diferentes temperaturas de aclimatación $\left(14,17\right.$ y $\left.22^{\circ} \mathrm{C}\right)$ con el fin de estimar la temperatura óptima y los límites térmicos de tolerancia de esta especie. La preferencia fue determinada mediante el método de temperatura preferida aguda. La tolerancia fue estimada mediante la temperatura crítica máxima y la temperatura letal media. Los resultados muestran que temperaturas de aclimatación mayores a $14^{\circ} \mathrm{C}$ incrementan la preferencia y tolerancia térmica de la especie. Sin embargo, individuos aclimatados a $22^{\circ} \mathrm{C}$ prefieren temperaturas menores a su aclimatación y limitan su tolerancia con respecto a los individuos aclimatados a $17^{\circ} \mathrm{C}$. La temperatura óptima estimada para juveniles de $\mathrm{A}$. scapularis fue de $18,7 \pm 2^{\circ} \mathrm{C}$ y su límite térmico superior e inferior de tolerancia fue de 32,6 y $10,1^{\circ} \mathrm{C}$, respectivamente. En base a los resultados se concluye que una temperatura de $18,7 \pm 2^{\circ} \mathrm{C}$ optimizaría los procesos fisiológicos en juveniles de $\mathrm{A}$. scapularis, y que temperaturas mayores a $32,6^{\circ} \mathrm{C}$ y menores a $10,1^{\circ} \mathrm{C}$ ocasionarían la muerte inmediata del organismo.
\end{abstract}

Palabras clave: Anisotremus scapularis, preferencia térmica, tolerancia térmica, temperatura de aclimatación, temperatura óptima

\section{INTRODUCCIÓN}

La chita Anisotremus scapularis (Tschudi, 1846), perteneciente a la familia Haemulidae, es un pez marino costero que se encuentra ampliamente distribuido desde la zona tropical en Manta $\left(0^{\circ} \mathrm{S}\right.$, Ecuador $)$, incluyendo islas Cocos, islas Galápagos y zonas influidas por el afloramiento costero peruano-chileno, hasta la zona templada en Antofagasta $\left(23^{\circ} \mathrm{S}\right.$, Chile) (Chirichigno \& Vélez 1998). Esta especie, también conocida como el roncador peruano (Peruvian Grunt en inglés), tiene importancia comercial para la pesquería artesanal del Perú alcanzando desembarques anuales de hasta 274 TM
(PRODUCE 2015). La demanda de chita en la costa peruana ha generado una necesidad por regular su pesquería artesanal, siendo su talla mínima de captura de $24 \mathrm{~cm}$ (PRODUCE 2016). Además; en vista de sus propiedades culinarias, su demanda y alto costo en el mercado ha sido considerada como especie de interés para la acuicultura (PRODUCE 2009).

El género Anisotremus comprende 10 especies descritas que se encuentran predominantemente en los arrecifes de coral y en los arrecifes rocosos subtropicales de los Neotrópicos del Pacífico Este, el Caribe y las aguas adyacentes (Bernardi et al. 
2008). Se han realizado estudios filogenéticos en especies de este género (Bernardi et al. 2008) y estudios de comunidades de peces de arrecifes en donde se registra información del nivel trófico en Anisotremus virginicus, A. moricandi, A. surinamensis, A. interruptus y A. taeniatus (Floeter et al. 2006, Grüss et al. 2016, Pastro et al. 2017, Sánchez et al. 2017). Así mismo, se conocen registros patológicos (Retinoblastoma) en A. virginicus (Reimschuessel et al. 1989) y también aspectos pesqueros (desembarques) de $A$. interruptus y A. davidsonii en el Golfo de California y en Baja California, respectivamente (Erisman et al. 2010, Shester \& Micheli et al. 2011). En este contexto, existe un desconocimiento de aspectos fisiológicos y de zootecnia en especies del género Anisotremus, a pesar de ser un género de alta importancia comercial y tener algunas especies de ornato (Tavera 2006).

Actualmente se han realizado esfuerzos de investigación sobre A. scapularis; lográndose su reproducción en cautiverio, descripción de su desarrollo ontogenético (IMARPE 2015a) y avances en su nutrición (Dionicio et al. 2016). Otros estudios se han centrado en mejorar el transporte de los juveniles de chita (Rosado et al. 2016) y en la descripción de los parásitos asociados a esta especie (Iannacone \& Alvariño 2012). Sin embargo, debido al estado incipiente de los estudios en relación a la zootecnia de A. scapularis, la información sobre las condiciones ambientales óptimas de cultivo para esta especie es aún desconocida.

La temperatura del agua es un factor ambiental importante, sobre todo, en los ectotermos acuáticos como los peces (Brett 1971, Reyes et al. 2011). Esta afecta todos los procesos metabólicos-fisiológicos de estos organismos influyendo consecuentemente sobre su desarrollo y sobrevivencia (Ficke et al. 2007) así como su abundancia y distribución geográfica (Terblanche et al. 2011). Al respecto, los peces pueden tolerar un rango de temperaturas que está limitado por temperaturas críticas. Los limites podrían responder a un colapso a nivel de la respiración celular de estos organismos (Pörtner 2010) afectando metabólicamente al organismo y conllevando a la muerte súbita a temperaturas superiores o inferiores a las críticas para una especie.

La teoría de la tolerancia térmica limitada por la capacidad de recepción de oxígeno de un organismo señala que dentro de esta hay un rango estrecho de temperaturas que optimizan los procesos fisiológicos (Pörtner \& Peck 2010). Estudios de preferencia térmica usan el comportamiento termoregulatorio de los peces para estimar su temperatura óptima que promueva su máximo crecimiento (Jobling 1981, Ward et al. 2010). A su vez, la temperatura crítica y la temperatura letal media son los indicadores más adecuados para estudiar la tolerancia térmica en peces (Somero 2010, Richard et al. 2012). Ante el desconocimiento de las condiciones óptimas de cultivo de $A$. scapularis y las variaciones térmicas del ambiente marino, conocer la preferencia y tolerancia térmica de esta especie es clave para obtener un mejor rendimiento del organismo en un eventual cultivo y comprender los posibles efectos de las variaciones térmicas sobre la captura de esta especie.

El presente trabajo evaluó la preferencia y tolerancia térmica de juveniles de A. scapularis a diferentes temperaturas de aclimatación, esto con el fin de estimar la temperatura óptima y los límites térmicos de tolerancia de juveniles de A. scapularis. Los resultados de este estudio están orientados a definir las condiciones para la optimización del cultivo de esta especie en fase juvenil, cuando los procesos bioenergéticos están orientados al crecimiento del organismo (Enberg et al. 2008). Los resultados obtenidos facilitarán la identificación de zonas marinas con potencial para el desarrollo de su cultivo y la definición de temperaturas de manejo en laboratorio. Así mismo, se brinda una visión sobre la vulnerabilidad de A. scapularis ante la variabilidad ambiental registrada en las costas de Perú.

\section{MATERIALES Y MÉTODOS}

\section{Material biológico}

Juveniles de Anisotremus scapularis (99 individuos) con un peso y longitud total promedio de 2,69 $\pm 0,12$ g y 5,60 \pm $0,09 \mathrm{~cm}$ (prom. $\pm \mathrm{ds}$ ), obtenidos en cautiverio en el Instituto del Mar del Perú (IMARPE), fueron trasladados al Laboratorio de Ecofisiología Acuática (IMARPE). Los peces fueron distribuidos equitativamente en 3 tanques de $2.000 \mathrm{~L}$ cada uno y en agua de mar a temperatura de $17^{\circ} \mathrm{C}$. La temperatura en un tanque fue disminuida a $14^{\circ} \mathrm{C}$ y en otro tanque aumentada a $22^{\circ} \mathrm{C}$ con una bomba de calor a una tasa de $1^{\circ} \mathrm{C} \cdot$ día $^{-1}$ hasta lograr temperaturas de 14,17 y $22^{\circ} \mathrm{C}$, respectivamente. Los peces fueron aclimatados a estas temperaturas por un periodo de 90 días previo a las evaluaciones experimentales.

Durante la aclimatación los peces fueron mantenidos con aireación (80-100\% de saturación de oxígeno). La concentración de oxígeno y la temperatura del agua se registraron con un oxímetro (HATCH-HQ40d). El fotoperiodo fue de $10 \mathrm{~h}$ luz por $14 \mathrm{~h}$ oscuridad. La alimentación fue realizada 2 veces al día ( 8 y 14 h) ad libitum con alimento comercial balanceado de marca Otohime: pellets extruídos del tamaño de $2 \mathrm{~mm}$ y $48 \%$ de proteína. El alimento residual y heces fueron removidos diariamente. Asímismo, se realizó un recambio diario de agua del $30 \%$ en cada tanque. La calidad del agua en los tanques fue mantenida por filtros mecánicos y biológicos asociados al sistema de recirculación de cada tanque. Previo a 


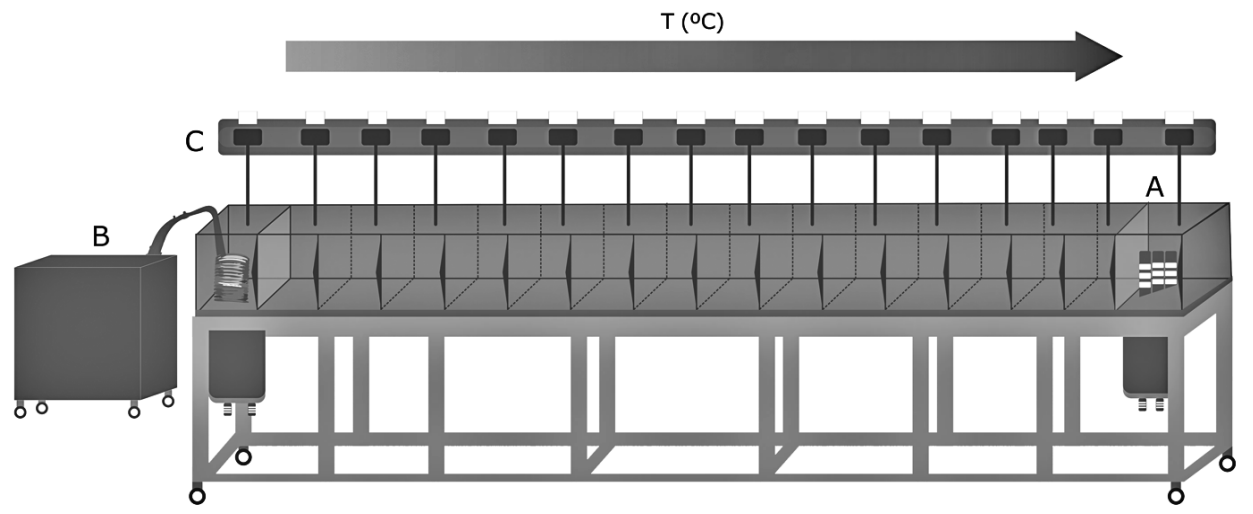

Figura 1. Representación esquemática del sistema de gradiente térmico horizontal utilizado para la determinación de la preferencia térmica. A) Calentador, B) Enfriador y C) Termómetros digitales / Schematic representation of the horizontal thermal gradient system used for the determination of thermal preference. A) Heater, B) Cooler and C) Digital thermometers

las pruebas de preferencia y tolerancia, los peces estuvieron en ayuno por $24 \mathrm{~h}$ para evitar que el incremento de la tasa metabólica y actividad del organismo afecten los valores de preferencia y tolerancia térmica (Reyes et al. 2011, Rodríguez 2014).

\section{Preferencia térmica}

Para determinar la temperatura óptima de juveniles de $A$. scapularis se realizó la prueba de la temperatura preferida aguda (TPA) (Reynolds \& Casterlin 1979). Esta prueba evaluó la temperatura de preferencia de los juveniles de A. scapularis en relación a su temperatura de aclimatación (con 5 réplicas para cada una de ellas). Para cada temperatura de aclimatación se usaron 4 individuos de similar tamaño dentro de un gradiente térmico horizontal regulado de 9 a $29^{\circ} \mathrm{C}$ (Fig. 1). El montaje del sistema incluyó un acuario de acrílico de 3,58 m de largo, $0,27 \mathrm{~m}$ de alto y $0,29 \mathrm{~m}$ de ancho con 3 calentadores de titanio (de $800 \mathrm{~W}$ cada uno) y un enfriador (de 1/3HP de potencia) colocados en los extremos opuestos del canal para crear el gradiente térmico horizontal. Este gradiente fue dividido virtualmente en 14 celdas térmicas, con un termómetro digital en cada una de ellas. Para evitar la estratificación térmica y mantener una concentración de oxígeno disuelto superior al $80 \%$ de saturación fue colocada una manguera de aireación a lo largo del gradiente (Fig. 1).

En el gradiente térmico 4 especímenes de $A$. scapularis, previamente anestesiados con tricaína $\left(0,108 \mathrm{~g} \cdot \mathrm{l}^{-1}\right)$, se colocaron enjaulados en la celda con la misma temperatura de aclimatación de procedencia. Una vez terminado el efecto de la tricaína, la jaula fue retirada para el acondicionamiento de los peces al gradiente durante 1 hora. Luego del acondicionamiento, se registró la posición de los individuos en las diferentes celdas y la temperatura en cada una de ellas cada 10 min durante las siguientes 3 horas. El registro de la posición de los peces fue realizado mediante una cámara de video HD (HALION ${ }^{\circledR} 12$ $\mathrm{mpx}$ ) conectada a una computadora. Este sistema de video fue ubicado detrás de una cortina azul eficiente para aislar al gradiente térmico y evitar que los ejemplares sean perturbados.

La temperatura de preferencia para cada réplica fue calculada como la mediana de la distribución de los datos de selección térmica (Jobling 1981). A estos valores de preferencia para cada temperatura de aclimatación se les realizó una regresión lineal con su respectivo intervalo de confianza. La temperatura óptima fue calculada según Fry (1947), en donde se define como 'aquella temperatura en la que la preferencia y la de aclimatación son iguales'. Bajo este concepto, la temperatura óptima fue determinada mediante una intersección entre la regresión lineal de la temperatura de preferencia en función de su aclimatación con una recta de $45^{\circ}$ de pendiente y origen de abscisas en cero (Fry 1947, Jobling 1981). La intersección entre las rectas indicó el punto igualdad entre la temperatura de preferencia y la de aclimatación. Sin embargo, este valor no es puntual y se representa mejor en un rango de temperaturas, para ello se realizó la intersección entre el intervalo de confianza de la regresión con la misma recta de $45^{\circ}$. Los datos fueron procesados con el programa estadístico $\mathrm{R}$ versión 3.2.1 ( ${ }^{\oplus}$ The R Foundation) ${ }^{1}$.

${ }^{1}$ The R Project for Statistical Computing. <https://www.r-project.org/> 


\section{TOLERANCIA TÉRMICA}

\section{TeMPeratura CRÍ́tICA MÁXIMA}

La prueba de temperatura crítica máxima (TCMáx) consistió en exponer a individuos de $A$. scapularis a un incremento de la temperatura (a una tasa constante de $1^{\circ} \mathrm{C} \cdot \mathrm{min}^{-1}$ ) hasta que el estado de pérdida de equilibrio haya sido alcanzado por el organismo (Lutterschmidt \& Hutchison 1997, Beitinger et al. 2000, Mora \& Maya 2006). La TCMáx fue determinada para cada temperatura de aclimatación con 11 réplicas de 1 individuo cada una. Se utilizó un sistema de incremento dinámico de la temperatura, el cual consistió de un acuario $(30$ x 70 × 30 $\mathrm{cm})$ con $54 \mathrm{~L}$ de agua de mar equipado con 5 calentadores (sumando $2.050 \mathrm{~W}$ ) sujetos a una piedra difusora de aire como medio de homogenización. El incremento de la temperatura en el acuario, por cada minuto, fue medido con un multiparámetro. Luego de alcanzado el estado de pérdida de equilibrio se procedió a evaluar la supervivencia de los peces durante $24 \mathrm{~h}$ en un sistema de recuperación dentro del tanque de cultivo.

La TCMáx para cada temperatura de aclimatación fue calculada como la media de las temperaturas en las que se alcanza la pérdida de equilibrio (Reyes et al. 2011). Para evaluar el efecto de la temperatura de aclimatación sobre la TCMáx se utilizó el análisis de Kruskal-Wallis $(\alpha=0,05)$. Finalmente, se realizó una prueba post-hoc de comparación múltiple (Dunntest, $\alpha=0,05)$ para evidenciar los grupos con diferencias significativas. Los datos fueron procesados con el programa estadístico R (versión 3.2.1).

\section{Temperatura LeTAL MEDIA}

Para determinar la temperatura letal media (TL50) superior e inferior se siguió el método planteado por Richard et al. (2012). Este método determina la temperatura a la cual se alcanza la muerte del $50 \%$ de un grupo de individuos ectotérmicos en un sistema de incremento o disminución de la temperatura a una tasa constante. Para la prueba se usó 20 juveniles de chita aclimatados a $16^{\circ} \mathrm{C}$ por 5 días (Schmidt-Nielsen 1990), la cual representa la temperatura promedio de la superficie del mar del sistema de afloramiento costero del Perú (Richard et al. 2012). Estos peces fueron repartidos equitativamente entre un sistema de incremento dinámico de la temperatura y un sistema de disminución de esta, ambos a $16^{\circ} \mathrm{C}$ y con una tasa de $1{ }^{\circ} \mathrm{C}$. día ${ }^{-1}$. El sistema de incremento de temperatura constó de un tanque con $250 \mathrm{~L}$ de agua de mar y dos calentadores (sumando $450 \mathrm{~W}$ ). Mientras que el sistema de disminución de esta constó de un tanque con $138 \mathrm{~L}$ de agua de mar y un enfriador (de $1 / 3$ HP de potencia). Durante las pruebas los peces se alimentaron 1 vez al día con Otohime, para registrar el estado de apetito. El registro continuo de la temperatura fue realizado por un sistema data-logger (T-QUIPU) basado en lenguaje y sistema Arduino.

\section{Resultados}

\section{TEMPERATURA PREFERIDA AGUDA}

La preferencia térmica de los juveniles de Anisotremus scapularis aumentó ligeramente conforme su temperatura de aclimatación aumentó. Éstas fueron 15,68 $\pm 1,27 ; 17,94 \pm 1,33$ y $20,23 \pm 0,99^{\circ} \mathrm{C}$ (promedio \pm desviación estándar) para la aclimatación a 14,17 y $22^{\circ} \mathrm{C}$, respectivamente. Se encontró una relación lineal positiva significativa $(P<0,01)$ entre los valores de la temperatura de preferencia y de aclimatación de la forma $\mathrm{y}=0,5612 \mathrm{x}+8,0449$ con un $\mathrm{r}^{2}=0,697$ (Fig. 2). La temperatura óptima fue estimada en $18,4^{\circ} \mathrm{C}$ dentro del rango entre 16,7 a $20,7^{\circ} \mathrm{C}$, dado por la intersección entre el intervalo de confianza de la regresión y la línea de $45^{\circ}$ (Fig. 2).

\section{Temperatura CRÍtiCa MáXima}

Los valores promedio de TCMáx de juveniles de A. scapularis, determinados con una tasa de $1{ }^{\circ} \mathrm{C} \cdot \mathrm{min}^{-1}$, fueron 29,$4 ; 32,6$ y $32,2^{\circ} \mathrm{C}$ para las temperaturas de aclimatación de 14,17 y $22^{\circ} \mathrm{C}$, respectivamente. La TCMáx incrementó en $3,14^{\circ} \mathrm{C}$ conforme aumentó la temperatura de aclimatación de 14 a $17^{\circ} \mathrm{C}$. Sin embargo, de 17 a $22^{\circ} \mathrm{C}$ no se observó una tendencia definida (Fig. 3). La supervivencia obtenida en los tres tratamientos fue del 80\%. Un análisis de Kruskal-Wallis indicó que la temperatura de aclimatación tuvo un efecto significativo $(\mathrm{H}=22,47$; $\mathrm{gl}=2$; $P<0,001)$ en la TCMáx, y a través de un análisis de comparación múltiple (Dunn-test) se determinó que existieron diferencias significativas de TCMáx entre los individuos aclimatados a 14 y $17^{\circ} \mathrm{C}(\mathrm{Z}=-4,53 ; P<0,001)$ y entre los de 14 y $22^{\circ} \mathrm{C}(\mathrm{Z}=-3,47 ; P<0,001)$. La temperatura de $32,6^{\circ} \mathrm{C}$ fue el mayor límite térmico superior observado para esta especie aclimatada en un intervalo de temperatura de 14 a $22^{\circ} \mathrm{C}$.

\section{Temperatura LETAL MEDIA}

En la prueba de la TL50 superior para juveniles de $A$. scapularis, aclimatados a $16^{\circ} \mathrm{C}$, se observó que en el intervalo térmico de 16 a $30^{\circ} \mathrm{C}$ hay condición de apetito. Además se evidenció que entre 27 y $30^{\circ} \mathrm{C}$ hay un aumento pronunciado de la actividad, siendo una temperatura de $31,7^{\circ} \mathrm{C}$ la desencadenante de la muerte del $50 \%$ de los individuos. Con respecto a la TL50 inferior se evidenció que a partir de una temperatura de $14^{\circ} \mathrm{C}$ ya no se registró la condición de apetito y que de $12 \mathrm{a} 11^{\circ} \mathrm{C}$ los peces empezaron a presentar un nado inclinado, siendo una temperatura de $10,1^{\circ} \mathrm{C}$ la que generó la muerte del $50 \%$ de los peces. 


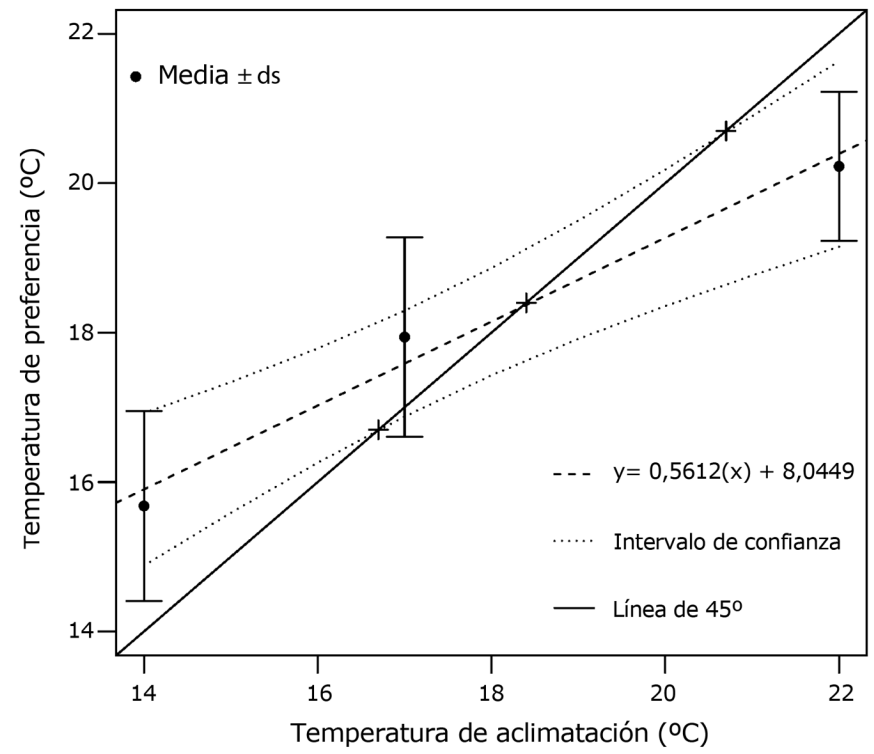

Figura 2. Temperatura de preferencia de juveniles de A. scapularis aclimatados a diferentes temperaturas. Los puntos negros representan la media de los valores de preferencia térmica para cada tem peratura de aclimatación. La regresión lineal entre ambas tem peraturas es representada por una línea discontinua. Las líneas punteadas representan el intervalo de confianza del $95 \%$ de la regresión lineal. La línea continua representa la línea de igualdad de $45^{\circ}$. Las cruces representan la temperatura a la cual la tem peratura preferida y la temperatura de aclimatación son la misma / Preferred temperature of A. scapularis juveniles acclimated to different temperatures. The black points represent the mean of the values of preferred temperature to each acclimation temperature. The linear regression between both temperatures is representing by the dashed line. The dotted lines represent the $95 \%$ confidence interval of the linear regression. The continuous line represents to the equality line of $45^{\circ}$. The crosses represent the temperature at which the preferred temperature and acclimation temperature are the same

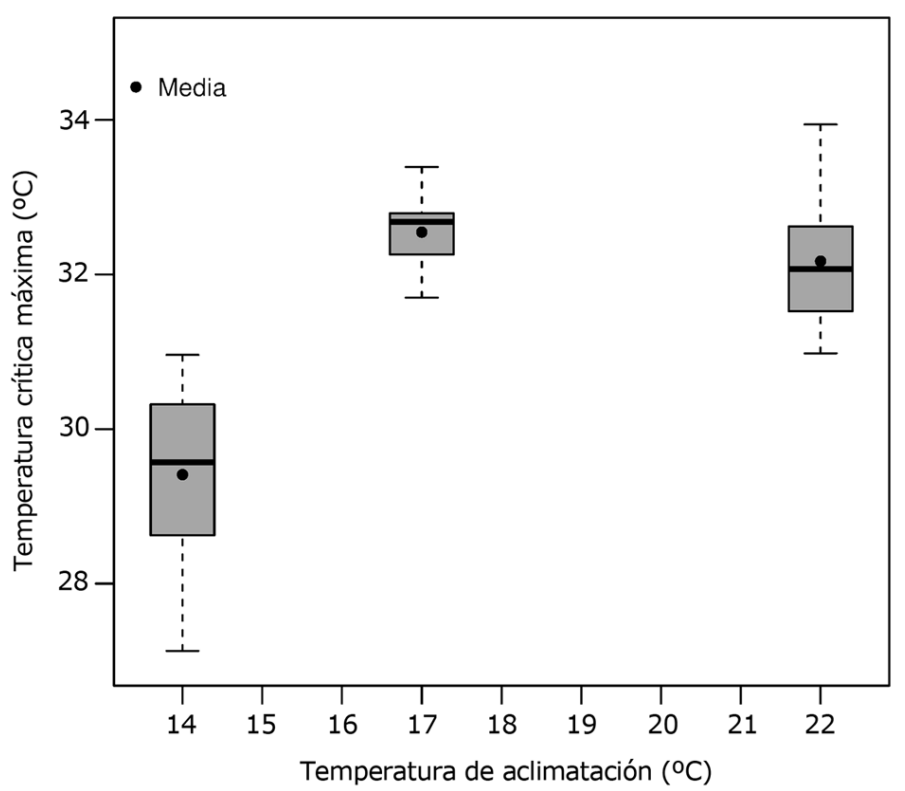

Figura 3. Temperatura crítica máxima de juveniles de A. scapularis aclimatados a diferentes temperaturas. Los puntos negros representan la media de los valores de la temperatura crítica máxima para cada temperatura de aclimatación / Critical thermal maximum of A. scapularis juveniles acclimated to different temperatures. The black points represent the mean of the values of the critical thermal maximum for each acclimation temperature 


\section{Discusión}

\section{Temperatura de PREFERENCIA}

Los peces presentan una variedad de relaciones preferenciatemperatura; éstas pueden ser categorizadas en 3 clases en base a si son funciones positivas, independientes o negativas frente a la temperatura de aclimatación (Johnson \& Kelsch 1998). La función positiva encontrada en juveniles de chita (Anisotremus scapularis) respondería a los ajustes fisiológicos compensatorios que permiten la optimización del metabolismo a una nueva temperatura (Kelsch \& Neill 1990). La capacidad de realizar estos ajustes compensatorios que incrementan la preferencia térmica en función de su aclimatación es propia de peces adaptados a variaciones térmicas graduales y prolongadas a lo largo del año, típicas de climas templados (Johnson \& Kelsch 1998). Esto se debe a que estas especies tienen el tiempo necesario para hacer cambios metabólicos que resulten en cambios sustanciales tanto en temperaturas de preferencia así como de tolerancia. Los juveniles de chita usados en este estudio provienen de individuos silvestres adaptados a la costa peruana, zona tropical con un afloramiento costero que por sus implicancias en la disminución de la temperatura superficial del mar podría haber determinado esta tendencia positiva propia de especies de climas templados.

El metabolismo de los peces está en función de la temperatura (Verberk et al. 2016). Ésta define el alcance metabólico aeróbico que satisface las demandas energéticas asociadas con el desarrollo de los organismos (Fry \& Hart 1948, Verberk et al. 2016). Los peces prefieren temperaturas que maximicen su alcance metabólico aeróbico, siendo éstas congruentes con la temperatura que optimiza sus procesos fisiológicos (Fry 1947, Kelsch 1996). Esto último es debido a que este alcance máximo permite que una gran cantidad de energía sea invertida en la optimización de procesos tales como el crecimiento y la reproducción (Blier et al. 1997, Clark et al. 2013). La relación entre el alcance metabólico aeróbico máximo y el mayor crecimiento ha sido demostrada para peces de importancia económica como el salmón rojo Oncorhynchus nerka (Brett 1976), el bacalao del atlántico Gadus morhua (Claireaux et al. 2000) y en hapuku Polyprion oxygeneios (Khan et al. 2014). En este contexto, la preferencia térmica puede ser usada como una medida que estima la temperatura óptima para los procesos fisiológicos (Kelsch \& Neill 1990) y también como un índice de temperatura a la cual las especies están adaptadas (Johnson \& Kelsch 1998).

La temperatura óptima obtenida en este estudio de 18,4 \pm $2{ }^{\circ} \mathrm{C}$ es respaldada por el mayor crecimiento en talla y peso que obtuvo Dionicio et al. $(2016)^{2}$ en esta especie, donde concluye que el mejor crecimiento es observado a 17 y $22^{\circ} \mathrm{C}$ con respecto a la temperatura de $14^{\circ} \mathrm{C}$. Por otro lado, la captura de chita en puertos pesqueros del Perú desde el 2004 al 2013 indica que hay una mayor captura de este recurso en Lambayeque, norte del Perú, alcanzándose durante este tiempo una captura acumulada de 267 TM (Oficina de Pesca Artesanal del IMARPE, com. pers.). En Lambayeque, el promedio mensual de la temperatura superficial del mar varía de 17 a $22^{\circ} \mathrm{C}$ a lo largo del año (IMARPE 2015b). Estos registros de captura y de temperatura concuerdan con el rango de la temperatura óptima obtenida en este estudio, ya que son indicadores que permiten conocer las preferencias térmicas de la especie y donde eventualmente se concentra el recurso.

\section{TOLerancia TÉRMICA}

Exposiciones de un organismo ectotérmico frente a temperaturas altas y bajas implican un incremento y diminución del metabolismo, respectivamente (Johnston \& Dunn 1987, Verberk et al. 2016). Sin embargo, este incremento y disminución tienen un límite térmico dado por las temperaturas críticas (máxima y mínima). Este efecto limitante se podría explicar por el concepto de la tolerancia térmica limitada por su capacidad de recepción de oxígeno (Frederich \& Pörtner 2000, Pörtner \& Peck 2010). Este concepto señala que la capacidad de tolerar un rango de temperaturas responde a la capacidad limitada de recepción de oxígeno del organismo, el cual podría deberse a la falla del sistema cardio-respiratorio a temperaturas críticas (Frederich $\&$ Pörtner 2000). Este fallo en el suministro y transporte de $\mathrm{O}_{2}$ ocasiona que se active la ruta anaeróbica para la obtención de energía que permita la supervivencia del organismo por un corto periodo de tiempo (Pörtner 2010). En este sentido, temperaturas más allá de las críticas dan como resultado la muerte inmediata del organismo (Pörtner \& Peck 2010).

La diferencia de la TCMáx de los juveniles de $A$. scapularis aclimatados en $17^{\circ} \mathrm{C}\left(32,6^{\circ} \mathrm{C}\right)$ o en $22^{\circ} \mathrm{C}\left(32,2^{\circ} \mathrm{C}\right)$ con respecto a los aclimatados a $14^{\circ} \mathrm{C}\left(29,4^{\circ} \mathrm{C}\right)$ podría ser fundamentada por los ajustes fisiológicos compensatorios del organismo (ajustes a nivel de membranas, enzimas y de proteínas) propios de cada temperatura de aclimatación (Kelsch \& Neill 1990). Estos ajustes podrían haber ocasionado que el fallo del sistema cardio-respiratorio se origine a diferentes temperaturas críticas (Pörtner \& Peck 2010). Al respecto, la capacidad de realizar estos ajustes compensatorios para cada temperatura de aclimatación, característicos de especies adaptadas a climas templados (como se explica en la sección de preferencia térmica), podrían ser debido a la adaptación de los juveniles de chita a las variaciones térmicas de la costa

2Dionicio J, R Galecio, S Fernando \& A Aguirre. 2016. Modelamiento del efecto de la temperatura sobre el crecimiento y consumo de oxigeno de juveniles de Anisotremus scapularis en laboratorio [Resumen]. Ciclo de Conferencias de Ecología Marina Cuantitativa. pp. 17. IMARPE. <http://www.imarpe.pe/imarpe/archivos/LibroResumenesFinal.pdf> 
peruana, las cuales son influidas por el sistema de afloramiento costero.

La zona marino costera del Perú frecuentemente experimenta importantes variaciones térmicas debido a eventos El Niño Oscilación del Sur (ENOS) y a la influencia del sistema de afloramiento costero (Aguirre et al. 2016). El sistema de afloramiento costero del Perú (provincia peruano-chilena) exhibe una variabilidad estacional e interanual de la temperatura. Durante el ciclo anual, las aguas de afloramiento (aguas frías) dominan la costa peruana; alcanzando su mayor amplitud en el invierno. A escala interanual, estas mismas aguas amplían su cobertura espacial durante La Niña; en tanto que durante El Niño estas aguas se repliegan o colapsan (Gutiérrez et al. 2011), incrementando así la temperatura superficial del mar. Esta variabilidad térmica que distingue a la costa peruana (Gárate \& Pacheco 2004, Chávez \& Messié 2009) podría explicar los cambios de tolerancia térmica respecto a su temperatura de aclimatación en juveniles de A. scapularis, pese a ser una especie de una zona tropical.

Al respecto, Gárate \& Pacheco (2004) registraron que después del evento El Niño 97-98 las especies de $A$. scapularis (provincia peruano-chilena) y A. interruptus (provincia panameña-aguas cálidas) coexistieron por un periodo en la bahía de San Bartolo, Perú. Esto debido a la amplitud de la distribución de A. interruptus y a la capacidad de aclimatación de A. scapularis. Ésta última afianzó la idea de que las especies del sistema de afloramiento costero del Perú (A. scapularis) podrían haber evolucionado bajo las condiciones de la variabilidad ocasionada por los eventos de El Niño y La Niña, tal como lo sugieren Arntz \& Tarazona (1988).

La limitación de la TCMáx entre la aclimatación a 17 y $22^{\circ} \mathrm{C}\left(32,6-32,2^{\circ} \mathrm{C}\right)$ se puede atribuir a que los individuos aclimatados a temperaturas fuera del rango óptimo (18,7 \pm $2{ }^{\circ} \mathrm{C}$ ) no tienen las condiciones necesarias para tolerar mayores temperaturas que las obtenidas dentro de éste (Jobling 1981, Pörtner 2010). Esto sugiere que una temperatura de 32,6 \pm $0,48^{\circ} \mathrm{C}$ es el límite térmico superior que puede tolerar como máximo esta especie.

Considerando que tasas rápidas de calentamiento podrían dar como resultado valores erróneos de tolerancia térmica (Paladino et al. 1980); el límite térmico superior de tolerancia, estimado por la TCMáx a $1^{\circ} \mathrm{C} \cdot \mathrm{min}^{-1}$, también se estimó por la TL50 a $1{ }^{\circ} \mathrm{C} \cdot$ día $^{-1}$ (Richard et al. 2012). La tasa de $1{ }^{\circ} \mathrm{C}$. $\mathrm{min}^{-1}$ es recomendada por Lutterschmidt \& Hutchison (1997) por ser lo suficientemente rápida para evitar efectos de aclimatación y lo suficientemente lenta para evitar desfases entre la temperatura del agua y la temperatura corporal. Por otro lado, la tasa de $1^{\circ} \mathrm{C}$. día ${ }^{-1}$ implica una exposición prolongada del organismo a una nueva temperatura sin que éste se aclimate, ya que se necesita de 3 a 5 días para aclimatación parcial de peces de climas templados y tropicales (Schmidt-Nielsen 1990). Según los resultados, la TL50 superior $\left(31,7^{\circ} \mathrm{C}\right)$ es ligeramente menor al valor de la TCMáx determinada para la aclimatación a $17^{\circ} \mathrm{C}\left(32,6^{\circ} \mathrm{C}\right)$, con una diferencia de $0,9^{\circ} \mathrm{C}$. Dado que la diferencia es pequeña se puede inferir que el uso de una tasa de calentamiento de $1^{\circ} \mathrm{C} \cdot$ día $^{-1}$ determina resultados de tolerancia consecuentes a los obtenidos con una tasa de $1^{\circ} \mathrm{C} \cdot \min ^{-1}$, respaldando a nuestros valores obtenidos de TCMáx.

Al respecto, el límite térmico superior máximo de juveniles de A. scapularis $\left(32,6^{\circ} \mathrm{C}\right)$ podría explicar que el evento $\mathrm{El}$ Niño del 97-98 (durante y después) no impactó negativamente en las capturas de chita en la bahía de San Bartolo y en el puerto pesquero de Salaverry, Perú (Tresierra et al. 1999, Gárate \& Pacheco 2004). En contraste a esto, hasta el momento no se tiene registro si los eventos de La Niña en el ecosistema marino peruano han afectado a esta especie. Sin embargo, es poco frecuente que ante eventos de La Niña la temperatura disminuya por debajo de su límite térmico inferior $\left(10,1^{\circ} \mathrm{C}\right)$.

El rango de tolerancia térmica de A. scapularis (10,1$32,6^{\circ} \mathrm{C}$ ) nos brinda una visión de la vulnerabilidad ante las variaciones térmicas de la costa peruana. Sin embargo, es necesario resaltar que temperaturas entre los 11 a $12^{\circ} \mathrm{C}$ (observadas en la TL50 inferior) ocasionan una disminución pronunciada de la actividad del organismo y que temperaturas entre los 27 a $30^{\circ} \mathrm{C}$ (observadas en la TL50 superior) generan un aumento pronunciado de este. Así, estas temperaturas pueden afectar el metabolismo y a todos los procesos fisiológicos involucrados en su desarrollo y supervivencia prolongada del organismo.

En conclusión, la temperatura que optimizaría los procesos fisiológicos de juveniles de chita (A. scapularis) es de 18,7 \pm $2{ }^{\circ} \mathrm{C}$. Esto contribuirá a identificar potenciales zonas de cultivo en la costa peruana, así como a establecer su temperatura de manejo para cultivo en laboratorio. Por otro lado, temperaturas inferiores a $10,1^{\circ} \mathrm{C}$ y superiores a $32,6^{\circ} \mathrm{C}$ en juveniles de $A$. scapularis podrían ser las causantes de la muerte inmediata del organismo.

\section{Agradecimientos}

Esta publicación fue realizada con el apoyo del IRD - JEAI EMACEP (Ecología Marina Cuantitativa del Ecosistema de Afloramiento Peruano) y del PpR Ordenamiento y Desarrollo de la Acuicultura (DGIA-IMARPE). Un especial agradecimiento a Kathy Córdoba por su apoyo durante la fase experimental del estudio. 


\section{LiTERATURA CITADA}

Aguirre A, F Jean, G Thouzeau \& J Flye-Sainte-Marie. 2016. Effects of progressive hypoxia on oxygen uptake in juveniles of the Peruvian scallop, Argopecten purpuratus (Lamarck, 1819). Aquaculture 451:385-389.

Beitinger T, W Bennett \& R McCauley. 2000. Temperature tolerance of North American freshwater fishes exposed to dynamic changes in temperature. Environmental Biology of Fishes 58(3): 237-275.

Blier PU, D Pelletier \& JD Dutil. 1997. Does aerobic capacity set a limit on fish growth rate? Reviews in Fisheries Science 5(4): 323-340.

Brett JR. 1971. Energetic responses of salmon to temperature. A study of some thermal relations in the physiology and freshwater ecology of sockeye salmon (Oncorhynchus nerka). American Zoologist 11: 99-113.

Brett JR. 1976. Scope for metabolism and growth of sockeye salmon, Oncorhynchus nerka, and some related energetics. Journal of the Fisheries Research Board of Canada 33(2): 307-313.

Claireaux G, DM Webber, JP Lagardère \& SR Kerr. 2000. Influence of water temperature and oxygenation on the aerobic metabolic scope of Atlantic cod (Gadus morhua). Journal of Sea Research 44: 257-265.

Clark TD, E Sandblom \& F Jutfelt. 2013. Aerobic scope measurements of fishes in an era of climate change: respirometry, relevance and recommendations. Journal of Experimental Biology 216: 2771-2782.

Chávez F \& M Messié. 2009. A comparative analysis of eastern boundary upwelling ecosytems. Progress in Oceanography 83: 80-96.

Chirichigno N \& M Vélez. 1998. Clave para identificar los peces marinos del Perú, 302 pp. Publicación Especial, Instituto del Mar del Perú, Callao.

Dionicio J, M Rosado, J Flores \& A Aguirre. 2016. Avances y perspectivas sobre crecimiento, alimentación y fisiología de juveniles de chita Anisotremus scapularis. World Aquaculture Society, Annual Meeting, Lima-Perú.

Enberg K, E Dunlop \& C Jørgensen. 2008. Fish growth (Ecological models). Reference module in earth systems and environmental sciences. Encyclopedia of Ecology 1: 15641572. Academic Press, Amsterdam.

Erisman B, I Mascarenas, G Paredes, Y Sadovy, O Aburto \& P Hastings. 2010. Seasonal, annual, and long-term trends in commercial fisheries for aggregating reef fishes in the Gulf of California, Mexico. Fisheries Research 106: 279288.

Ficke AD, CA Myrick \& LJ Hansen. 2007. Potential impacts of global climate change on freshwater fisheries. Reviews in Fish Biology and Fisheries 17: 581-613.

Floeter S, B Halpern \& C Ferreira. 2006. Effects of fishing and protection on Brazilian reef fishes. Biological Conservation 128: 391-402.

Frederich M \& H Pörtner. 2000. Oxygen limitation of thermal tolerance defined by cardiac and ventilatory performance in spider crab, Maja squinado. American Journal of Physiology - Regulatory, Integrative and Comparative Physiology 279(5): R1531-R1538.
Fry FEJ. 1947. Effects of the environment on animal activity. University of Toronto Studies Biology Series 55, Publication of the Ontario Fisheries Research Laboratory 68: 1-62.

Fry FEJ \& JS Hart. 1948. The relation of temperature to oxygen consumption in the goldfish. The Biological Bulletin 94:66-77.

Gárate A \& A Pacheco. 2004. Cambios en la distribución y abundancia de la ictiofauna de aguas someras en San Bartolo (Lima, Perú) después del ENSO 1997-98. Ecología Aplicada 3(1-2): 148-153.

Grüss A, M Schirripa, D Chagaris, L Velez, Y-J Shin, P Verley, R Oliveros-Ramos \& C Ainsworth. 2016. Estimating natural mortality rates and simulating fishing scenarios for Gulf of Mexico red grouper (Epinephelus morio) using the ecosystem model OSMOSE-WFS. Journal of Marine Systems 154: 264-279.

Gutiérrez D, A Bertrand, C Wonitza-Mendo, B Dewitte, S Purca, C Peña, A Chaigneau, J Tam, M Graco, Vincent Echevin, C Grados, P Fréon \& R Guevara. 2011. Sensibilidad del sistema de afloramiento costero del Perú al cambio climático e implicancias ecológicas. Revista Peruana Geo-Atmosférica RPGA 3: 1-24.

Iannacone J \& L Alvariño. 2012. Microecology of the monogenean Mexicana sp. on the gills of Anisotremus scapularis (Tschudi, 1846) (Osteichthyes, Haemulidae) of the marine coast of Lima, Peru. Neotropical Helminthology $6(2): 277-285$.

IMARPE. 2015a. Ciclo de vida de la Chita: Anisotremus scapularis. Serie de Divulgación Científica 1(1). <http:// biblioimarpe.imarpe.gob.pe:8080/handle/123456789/3004>

IMARPE. 2015b. Monitoreo satelital diario de parámetros oceanográficos del mar peruano: Temperatura superficial del mar. Área funcional de sensoramiento remoto [base de datos en Internet]. Perú, Instituto del Mar de Perú, 2015. <http://satelite.imarpe.gob.pe/uprsig/sst_prov.html>

Jobling M. 1981. Temperature tolerance and the final preferendum - rapid methods for the assessment of optimum growth temperatures. Journal of Fish Biology 19: 439-455.

Johnston I \& J Dunn. 1987. Temperature acclimation and metabolism in ectotherms with particular reference to teleost fish. Symposia of the Society for Experimental Biology 41: 67-93.

Johnson JA \& SW Kelsch. 1998. Effects of evolutionary thermal environment on temperature-preference relationships in fishes. Environmental Biology of Fishes 53: 447-458.

Kelsch SW. 1996. Temperature selection and performance by bluegills: evidence for selection in response to available power. Transactions of the American Fisheries Society 112: 424430.

Kelsch SW \& WH Neill. 1990. Temperature preference versus acclimation in fishes: selection for changing metabolic optima. Transactions of the American Fisheries Society 119: 601610.

Khan JR, S Pether, M Bruce, SP Walker \& NA Herbert. 2014. Optimum temperatures for growth and feed conversion in cultured hapuku (Polyprion oxygeneios) - is there a link to aerobic metabolic scope and final temperature preference? Aquaculture 430: 107-113. 
Lutterschmidt WI \& VH Hutchison. 1997. The critical thermal maximum: History and critique. Canadian Journal of Zoology 75(10): 1561-1574.

Mora C \& MF Maya. 2006. Effect of the rate of temperature increase of the dynamic method on the heat tolerance of fishes. Journal of Thermal Biology 31: 337-341.

Paladino F, J Spotila, J Schubauer \& K Kowalski. 1980. The critical thermal maximum-a technique used to elucidate physiological stress and adaptation in fishes. Review of Canadian Biology 39: 115-122.

Pastro G, G Dias, G Pereira \& F Gibran. 2017. The consequences of small-scale variations in habitat conditions driven by a floating marina on reef fish assemblages of SE Brazil. Ocean \& Coastal Management 141: 98-106.

Pörtner HO. 2010. Oxygen - and capacity - limitation of thermal tolerance: a matrix for integrating climate-related stressor effects in marine ecosystems. Journal of Experimental Biology 213: 881-893.

Pörtner HO \& MA Peck. 2010. Climate change effects on fishes and fisheries: towards a cause-and - effect understanding. Journal of Fish Biology 77: 1745-1779.

PRODUCE. 2009. Plan Nacional de Desarrollo Acuícola 20102021. PRODUCE, Perú, Ministerio de la Producción, Lima. <http://www.produce.gob.pe/pesqueria/publicaciones/2010/ enero/ds001-2010-produce.pdf $>$

PRODUCE. 2015. Anuario estadístico pesquero y acuícola 2014. PRODUCE, Perú, Ministerio de la Producción. <http://www.produce.gob.pe/documentos/estadisticas/ anuarios/anuario-estadistico-pesca-2014.pdf>

PRODUCE. 2016. Resolución Ministerial Nº 156-2016PRODUCE, Establecimiento de la Talla Mínima de Captura (TMC) del recurso de chita Anisotremus scapularis, El Peruano, 27 de abril de 2016. PRODUCE, Perú, Ministerio de la Producción. <http://busquedas.elperuano. com.pe/normaslegales/establecen-la-talla-minima-decaptura-tmc-del-recurso-de-c-resolucion-ministerial-no-1562016-produce-1373780-1/>

Reimschuessel R, R Bennett, E May \& M Lipsky. 1989. Retinoblastoma in a porkfish (Anisotremus virginicus, Linnaeus) and a brown bullhead (lctalurus nebulosus, Lesuer). Journal of Comparative Pathology 101: 215-219.

Reyes I, F Díaz, AD Re \& J Pérez. 2011. Behavioral thermoregulation, temperature tolerance and oxygen consumption in the Mexican bullseye pufferfish, Sphoeroides annulatus Jenyns (1842), acclimated to different temperatures. Journal of Thermal Biology 36: 200-205.

Reynolds W \& M Casterlin. 1979. Behavioral thermoregulation and the final preferendum paradigm. American Zoologist 19: 211-224.

Richard J, SA Morley, MAS Thorne \& LS Peck. 2012. Estimating long-term survival temperatures at the assemblage level in the marine environment: Towards macrophysiology. PLoS ONE 7(4): e34655. <doi:10.1371/journal.pone. 0034655>
Rodríguez M. 2014. Efecto de la temperatura de aclimatación sobre el comportamiento térmico y de alimentación de los juveniles de jurel cola amarilla Seriola lalandi. Tesis de Maestro en Ciencias en Acuicultura, Programa de Posgrado en Ciencias en Acuicultura, Centro de Investigación Científica y de Educación Superior de Ensenada (CICESE), Baja California, $60 \mathrm{pp}$.

Rosado M, J Dionicio \& A Aguirre. 2016. Evaluación de diferentes concentraciones de Tricaína (MS-222) en el transporte de chita (Anisotremus scapularis) juveniles. Revista de Investigaciones Veterinarias del Perú 27(4): 687 697.

Sánchez C, J Borges, G De La Cruz \& S Ferse. 2017. Links between fish community structure and habitat complexity of a rocky reef in the Gulf of California threatened by development: Implications for mitigation measures. Ocean \& Coastal Management 137: 96-106.

Schmidt-Nielsen K. 1990. Animal physiology: adaptation and environment, 602 pp. Cambridge University Press, Cambridge.

Shester G \& F Micheli. 2011. Conservation challenges for small-scale fisheries: Bycatch and habitat impacts of traps and gillnets. Biological Conservation 144: 1673-1681.

Somero GN. 2010. The physiology of climate change: how potentials for acclimatization and genetic adaptation will determine 'winners' and 'losers'. Journal of Experimental Biology 213: 912-920.

Tavera J. 2006. Análisis filogenético y biogeográfico del género Anisotremus Gill, 1861 (Perciformes: Haemulidae). Tesis de Maestría en Manejo de Recursos Marinos, Instituto Politécnico Nacional, Centro Interdisciplinario de Ciencias Marinas, La Paz, México, 96 pp.

Terblanche JS, AA Hoffman, KA Mitchell, L Rako, PC le Roux \& LS Chown. 2011. Ecologically relevant measures of tolerance to potentially lethal temperatures. Journal of Experimental Biology 214:3713-3725.

Tresierra A, Z Culquichicón \& B Veneros. 1999. Efecto del fenómeno 'El Niño 1997-98' en el desembarque de los recursos pesqueros artesanales de puerto Salaverry (Trujillo). Revista Peruana de Biología 6(3): 160-163.

Verberk W, J Overgaard, R Ern, M Bayley, T Wang, L Boardman \& J Terblanche. 2016. Does oxygen limit thermal tolerance in arthropods? A critical review of current evidence. Comparative Biochemistry and Physiology, Part A 192: 64-78.

Ward AJW, EMA Hensor, MN Webster \& PJ Hart. 2010. Behavioral thermoregulation in two freshwater fish species. Journal of Fish Biology 76: 2287-2298. 